\section{Abstractions}

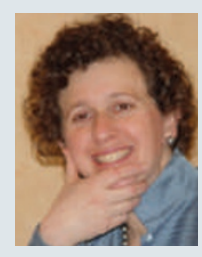

\section{FIRST AUTHOR}

Stars form in dense clouds of cold, turbulent molecular gas, but astronomers have had difficulty dissecting these clouds' complex structures to determine the mechanisms that drive star formation. On page 63, Alyssa Goodman, an astrophysicist at the HarvardSmithsonian Center for Astrophysics, and her colleagues adapt the dendrogram - a technique typically used to depict evolutionary relationships using a 'tree' diagram - to obtain a realistic representation of the internal structure of a molecular cloud. Goodman describes how her team created the first three-dimensional (3D) figure that can be manipulated by Nature readers.

\section{Was it difficult to show your data in three} dimensions?

We got lucky. At a conference in 2004, I spoke about how dissatisfied I was with the way astronomers typically deal with 3D data - by compressing them to $2 \mathrm{D}$ or showing a series of 'slices' through a cube. Afterwards, Michael Halle, a computer scientist at the Brigham and Women's Hospital in Boston, Massachusetts, said that his surgical-planning software, used to image organs ahead of and during surgery, could manipulate and analyse high-dimensional data and might solve my problems. Co-author Michelle Borkin, then an undergraduate student, tried it with our data, and a graphics company then helped us put the 3D pdfs together.

Why did you decide to use a dendrogram? We knew we needed a way to describe the interconnectedness - how certain 3D objects are nested within other 3D objects - of different cloud regions. Drawing a tree is a natural way to explain that kind of hierarchical relationship, but is rarely used in our field.

\section{Will your findings usher in a new era of research?}

Yes. Our work shows that an object's own gravity is an important force working, over millions of years and on multiple length scales, to shape a cloud's internal structure. Theoreticians typically ignore gravity in star-formation simulations until the final stages, when fluctuations previously caused by turbulence need to collapse in order for a star to form. But now simulators will need to include gravity earlier on.

Will this help to explain how stars acquire their mass?

It should, because now we can isolate selfgravitating - presumably star-forming gas from its more transient surroundings. At very high resolution, this procedure will soon allow us to directly compare the frequency distribution of self-gravitating gas mass with that of stellar mass.
MAKING THE PAPER

\section{Dan Barouch}

\section{Changing vaccine carrier renews promise of anti-HIV therapy.}

In research, there is always the risk that someone else will beat you to the finishing line. On top of that, there is the chance that another's findings might undermine your rationale. Dan Barouch of Harvard's Beth Israel Deaconess Medical Center was in the middle of a threeyear study testing adenovirus-based vaccine regimens in monkeys when a clinical trial of an HIV vaccine that also relied on an adenovirus failed. But Barouch, whose work centred on the related simian immunodeficiency virus (SIV), persevered, and now reports that his vaccine can protect the animals against infection. His results demonstrate that not all adenovirus vectors need be shelved.

In September 2007, Merck's phase II HIVvaccine trial, dubbed STEP, was called off because findings showed that it did not prevent HIV infection. The approach involved inserting snippets of HIV genes into a vector - a weakened version of a common-cold-causing adenovirus - which was then injected into human patients. The idea was that proteins produced by the adenovirus would stimulate production of immune-system cells called $\mathrm{T}$ cells, and that these would then recognize and kill HIV-infected cells.

The problem with the STEP study was that the kind of adenovirus used - adenovirus serotype 5 (Ad5) - is one of the most common of the 51 known human adenoviruses. As a result, says Barouch, when an Ad5 vector is injected into a person it is recognized by pre-existing antibodies. "In somebody who has pre-existing immunity, Ad5 antibodies may neutralize the vaccine vector before it can do its job," he explains. $\mathrm{He}$ reasoned that a way around the problem would be to use adenoviruses rarely found in humans.

Barouch's group thus sought to identify and develop rare-serotype adenovirus vectors. One

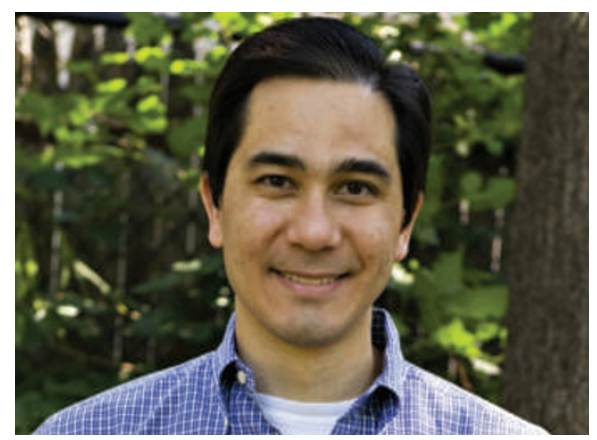

candidate, Ad26, is rare in US populations as well as in sub-Saharan Africa, and induced immune responses in animals. In Barouch's study, monkeys received a priming shot of an Ad26-based vaccine and then, several months later, a booster shot of an Ad5-based vaccine. This use of two adenoviruses made the interference of neutralizing antibodies less likely. When exposed to SIV, these monkeys ended up with fewer virus particles in their blood and had a lower risk of dying from infection than those that did not receive the vaccine. By contrast, regimens using Ad5 alone failed to protect the monkeys against SIV infection (see page 87 ).

That the Ad26/Ad5 regimen worked so well in a setting in which an Ad5-only regimen failed suggests to Barouch that STEP was not the end of the road for T-cell-based vaccines for HIV. His team's work is only a proof-of-concept study, he adds, but it shows that "improved T-cell-based vaccine regimens might be able to do better than the regimen that failed in the STEP study". And we may soon find out whether this is the case: the Ad26 vector is now in a phase I clinical trial.

The STEP study was so discouraging that some prominent AIDS researchers called for all funding of clinical vaccine research to be redirected to basic research. Barouch advocates a more balanced approach. Although the field of HIV-vaccine research certainly needs much more basic knowledge to solve fundamental scientific problems, he says, both basic research and clinical research should be pursued. "If we had not conducted the STEP study, we would be light years behind where we are today."

\title{
FROM THE BLOGOSPHERE
}

Nature Chemical Biology

associate editor Catherine Goodman treats readers of the Sceptical Chymist blog to a blow-by-blow account of a typical workday (http://tinyurl. com/5kuver). Here are some highlights:

8:53 I'm putting together an agenda for a meeting tomorrow, so that probably takes priority.

11:31 Debating the merits of a paper. Initial feeling is that sugars are cool. 12:28 Got an awesome review outline from an author ... Very excited! Also had to answer some questions about proofs and read a couple of papers as possible research highlights. 3:37 Hopefully commissioned a News \& Views. 4:02 Making some notes on the review outline from earlier. Also wrote back to someone who has a cool story they want to submit to us.

In her final entry at the end of the day, Goodman reflects that, despite having a productive day, she had not devoted enough time to reviewing manuscripts. "As a result, I'Il take some papers home to read overnight and plan to devote much more time to our submissions tomorrow," she says. 